\title{
Transmit Subaperturing for MIMO Radars with Nested Arrays *
}

\author{
Linlin $\mathrm{Mao}^{\dagger} \quad$ Hongbin $\mathrm{Li}^{\ddagger} \quad$ Qunfei Zhang $\S$
}

December 12, 2016

\begin{abstract}
We consider a multiple-input multiple-output (MIMO) radar equipped with a colocated nested array for both transmit and receive. A novel transmit subaperturing (TS) approach that decomposes the nested array into unequal subarrays is proposed to take the advantage of the degrees of freedom (DOF) provided by the nested array but without resorting to spatial smoothing. Phased-array and the conventional MIMO with omni-directional transmission (omni-MIMO) are also extended to the nested array scenario, which utilize spatial smoothing to improve their DOF. Simulation results show that with sufficient training data, the omni-MIMO with nested array achieves the highest output signalto-interference-plus-noise ratio (SINR), while the TS-MIMO scheme outperforms the other methods when training is limited when the radar operates in a non-homogeneous environment.

Keywords - Nested array (NA), subaperturing, transmit beamforming, degrees of freedom (DOF).

\section{Introduction}

Multiple-input multiple-output (MIMO) radar has been the subject of intensive research in recent years, due to the fact that it can extend the array aperture by virtual sensors and thereby provide higher spatial resolution, better parameter identifiability and more degrees of freedom (DOF) than its phased-array counterpart [1-8]. However, the advantages offered by MIMO radar come at the price of losing the directional gain and spatial selectivity offered by the phased-array radar. To overcome these disadvantages, intermediates between the MIMO and phased-array have been investigated to

\footnotetext{
${ }^{*}$ The work of H. Li was supported in part by the National Science Foundation under grant ECCS-1609393 and the Air Force Office of Scientific Research under grant FA9550-16-1-0243.

${ }^{\dagger}$ L. Mao is with the School of Marine Science and Technology, Northwestern Polytechnical University, Xi'an 710072, China. This work is completed during her visit to the Department of Electrical and Computer Engineering, Stevens Institute of Technology, Hoboken, NJ 07030 USA (e-mail: maple3511@nwpu.edu.cn).

${ }^{\ddagger} \mathrm{H}$. Li is with the Department of Electrical and Computer Engineering, Stevens Institute of Technology, Hoboken, NJ 07030 USA (e-mail: hongbin.li@stevens.edu).

${ }^{\S}$ Q. Zhang is with the School of Marine Science and Technology, Northwestern Polytechnical University, Xi'an 710072, China (e-mail: zhangqf@nwpu.edu.cn).
} 
seek benefits of both. Particularly, a transmit subaperturing (TS) approach was proposed in [9] for MIMO radar with a uniform linear array (ULA) to form multiple transmit beams steered toward the same direction in order to obtain a transmit array gain. The resulting system is referred to as the TS-MIMO radar. In [10,11], the authors proposed a phased-MIMO technique, which enjoys the advantages of MIMO without sacrificing the directional gain of phased-array. All these works divided the transmit array into subarrays of equal length. The authors of [12] presented a phased MIMO radar with unequal subarrays named HPMR-US, which divides the whole transmit array into overlapping and variable-sized partitions. It was shown that HPMR-US can provide a better directional gain than its equal-sized subarray counterpart. An extension of the transmit subaperturing strategy to frequency diverse arrays was considered for range and angle estimation in [13]. All the above works have focused on the case when ULA is employed for both transmit and receive.

Meanwhile, nested array (NA) constructed by combining two or more ULAs has recently received considerable attention due to its potential to increase the DOF [14-16]. Specifically, [14] proposed a NA based on the concept of difference coarray (DC), which can offer $O\left(N^{2}\right)$ DOF from only $N$ physical sensors. An extension of NA to the case involving vector-sensor arrays was investigated in [15]. Inspired by the phased-MIMO configuration [9] and [10], and the NA structure [14], a phasedMIMO system using a ULA for transmit and a different NA for receive was proposed in [17] to increase the DOF of the whole system.

In order to seek benefits such as additional DOF and more directional gain, we consider a MIMO radar using an NA for both transmit and receive. To the best of our knowledge, NA has not been explored for transmit in MIMO radar. Using an NA for both transmit and receive helps to expand the effective array aperture resulting from both sum coarray and DC processing. A novel transmit subaperturing approach with unequal subarrays is proposed to take the advantage of the DOF provided by the nested array without resorting to spatial smoothing. Phased-array and omni-MIMO transmission [9] are also extended to the NA scenario. The advantages of the proposed MIMO radar with NA for both transmit and receive are illustrated via numerical examples.

\section{Problem formulation}

Consider a colocated MIMO system consisting of $N$ antennas with a NA used for both transmit and receive. These $N$ antennas form an optimal two-level NA, which is a concatenation of an inner ULA with $J=\left\lfloor\frac{N}{2}\right\rfloor$ elements, and an outer ULA with $K=N-J$ elements [14]. The spacing between two adjacent elements of the inner array is $d=\lambda / 2$ (half wavelength), while the counterpart 
for the outer array is $J d$. The corresponding sensor locations are given by the union of the sets $S_{\text {in }}=\{j d, j=0,1, \cdots, J-1\}$ and $S_{\text {out }}=\{((J+1) k-1) d, k=1,2, \cdots, K\}$. Nested array provides an effective approach to increase the DOF at the receiver side. An optimal two-level NA attains $2 K(J+1)-1$ DOF in the coarray using only $J+K$ elements. It is noted that NA is a receive processing technique originally introduced for passive sensing. In this paper, we discuss new transmit approaches for NAs in active sensing and explore the associated benefits in terms of DOF and interference cancellation capability with limited training data.

\section{Proposed Schemes}

We introduce three different transmit schemes, which are extensions of those in [9] to the case of NAs. The extensions are non-trivial, especially for the transmit subaperturing scheme, due to the non-uniform structure of the nested array.

\subsection{Transmit Schemes}

\subsubsection{Transmit Subaperturing (TS) with Unequal Subarrays}

The main idea is to construct multiple subarrays of unequal sizes for transmission. On one hand, it is desirable to include large subarrays, since beamforming over large subarray offers large directional gain; on the other hand, it is also beneficial to have as many subarrays as possible, since each subarray transmits a different waveform, leading to larger DOF. Taking both into consideration, we propose the following design criteria:

(i) The subarrays should cover all possible spacings provided by the DC of an optimal two-level NA, namely $\{m d, m=1,2, \cdots, K(J+1)-1\} ;$

(ii) Each spacing is employed exclusively by only one subarray as its maximum spacing.

In general, a transmit subarray consists of a unique number of antennas ranging from 1 to $N$ such that no two subarrays have the same size. This is different from the traditional TS-MIMO [9] or phasedMIMO [10] system, which groups the array elements of a ULA into equal-sized transmit subapertures. Following the above criteria, one can come up with different forms of TS. Next, we present a simple construction.

Fig. 1 shows an illustrating example of the proposed TS scheme with unequal subarrays for $N=4$ antennas. A total of $M=K(J+1)-1=2 \times(2+1)-1=5$ subarrays are constructed, which cover all possible spacings from $d$ to $5 d$. These subarrays can be classified into 2 groups: 
- Group I has $J$ subarrays of aperture size $m d, m=1,2, \cdots, J$. The $m$ th subarray is a ULA with elements positions given by

$$
A_{m}=\{p d, p=0, \cdots, m\} .
$$

For the example shown in Fig. 1, Group I contains 2 subarrays given by $A_{1}=\{0, d\}$ and $A_{2}=$ $\{0, d, 2 d\}$.

- Group II consists of $(J+1)(K-1)$ subarrays of aperture size $m d, m=J+1, \cdots, K(J+1)-1$. The $m$ th subarray is an array with elements positions given by

$$
A_{m}=\left\{(p(J+1)-1) d, p=1,2, \cdots, \frac{m}{J+1}+1\right\}
$$

for $m \in\{q(J+1), q=1, \cdots, K-1\}$, and

$$
A_{m}=A_{m-1} \cup\left\{\left\lceil\frac{m}{J+1}\right\rceil(J+1)-1-m\right\}
$$

for $m \notin\{q(J+1), q=1, \cdots, K-1\}$. For the system in Fig. 1, Group II contains 3 subarrays given by $A_{3}=\{2 d, 5 d\}, A_{4}=\{d, 2 d, 5 d\}$, and $A_{5}=\{0, d, 2 d, 5 d\}$.

Following the above guideline, one can easily form transmit subapertures for any other NAs.

Assume that all the $M$ transmit subapertures are engaged in the TS transmission, each modulating a different waveform. Let $\mathbf{b}_{m}(\theta)$ denote the steering vector associated with the $m$ th transmit subaperture, which is an $\left|A_{m}\right| \times 1$ sub-vector extracted from the transmit steering vector $\mathbf{a}_{t}(\theta)$ for the entire array, where $\left|A_{m}\right|$ denotes the cardinality of $A_{m}$. For the configuration shown in Fig. $1, \mathbf{a}_{t}(\theta)$ and $\mathbf{b}_{4}(\theta)$ are:

$$
\begin{gathered}
\mathbf{a}_{t}(\theta)=\left[\begin{array}{llll}
1 & e^{-j \pi \sin \theta} & e^{-j 2 \pi \sin \theta} & e^{-j 5 \pi \sin \theta}
\end{array}\right] \\
\mathbf{b}_{4}(\theta)=\left[\begin{array}{lll}
e^{-j \pi \sin \theta} & e^{-j 2 \pi \sin \theta} & e^{-j 5 \pi \sin \theta}
\end{array}\right]
\end{gathered}
$$

On the other hand, the receive array employs one single aperture consisting of all $N$ elements. Suppose there is a target located at the look direction $\theta_{0}$ and $I$ uncorrelated interference sources located at look directions $\theta_{i} \neq \theta_{0}, i=1, \cdots, I$. By assuming orthogonal waveforms, matched filtering and vectorizing can be applied on the received signal, which gives [9]

$$
\mathbf{y}_{\mathrm{ts}}=\kappa \alpha_{0} \mathbf{a}_{\mathrm{ts}}\left(\theta_{0}\right)+\kappa \sum_{i=1}^{I} \alpha_{i} \mathbf{a}_{\mathrm{ts}}\left(\theta_{i}\right)+\mathbf{n}
$$

where $\alpha_{0}$ and $\alpha_{i}$ are the complex amplitudes of the target and the $i$ th interference after the matched filter, respectively, $\mathbf{n}$ denotes the additive Gaussian noise vector with zero mean and covariance matrix $\sigma_{n}^{2} \mathbf{I}$, and

$$
\mathbf{a}_{\mathrm{ts}}(\theta)=\kappa \boldsymbol{\zeta}(\theta) \otimes \mathbf{a}_{r}(\theta)
$$


denotes the $M N \times 1$ effective array manifold and $\mathbf{a}_{r}(\theta)$ the receive steering vector, which is the same as $\mathbf{a}_{t}(\theta)$ since the NA is shared for transmit and receive. $\zeta(\theta)$ is an $M \times 1$ vector with the $m$ th element given by $\zeta_{m}(\theta) \triangleq \mathbf{b}_{m}^{\mathrm{T}}(\theta) \mathbf{b}_{m}^{*}\left(\theta_{0}\right) . \kappa$ is an amplitude scaling parameter

$$
\kappa=\sqrt{\frac{N}{\sum_{m}\left|A_{m}\right|}}
$$

which is used to ensure identical total transmitted energy for different transmit schemes.

\subsubsection{Phased-Array}

For the NA radar system, a phased-array like transmission employs one transmit aperture consisting of all $N$ elements forming a directional transmit beam pointing toward some look direction $\theta_{0}$. The received signal is processed by a matched filter, which outputs

$$
\mathbf{y}_{\mathrm{pa}}=\alpha_{0} \mathbf{a}_{\mathrm{pa}}\left(\theta_{0}\right)+\sum_{i=1}^{I} \alpha_{i} \mathbf{a}_{\mathrm{pa}}\left(\theta_{i}\right)+\mathbf{n}
$$

The $N \times 1$ vector

$$
\mathbf{a}_{\mathrm{pa}}(\theta)=\mathbf{a}_{t}^{T}(\theta) \mathbf{a}_{t}^{*}\left(\theta_{0}\right) \mathbf{a}_{r}(\theta)
$$

is referred to as the joint transmit-receiving steering vector.

\subsubsection{Omni-Directional Transmission}

For the NA radar system, an omni-directional transmission employs all $N$ elements, each transmitting an orthonormal waveform. Applying matched filtering and vectorizing on the received signals yields

$$
\mathbf{y}_{\mathrm{od}}=\alpha_{0} \mathbf{a}_{\mathrm{od}}\left(\theta_{0}\right)+\sum_{i=1}^{I} \alpha_{i} \mathbf{a}_{\mathrm{od}}\left(\theta_{i}\right)+\mathbf{n}
$$

where

$$
\mathbf{a}_{\mathrm{od}}(\theta)=\mathbf{a}_{t}(\theta) \otimes \mathbf{a}_{r}(\theta)
$$

denotes the $N^{2} \times 1$ joint transmit-receive steering vector.

\subsection{Adaptive Beamforming}

We now consider adaptive beamforming for the three transmit schemes. For notational convenience, we drop subscripts "pa", "od" and "ts". The target and interference scatterers are assumed to be spatially distributed objects which are independent of each other. As a result, the target/interference complex amplitudes are mutually uncorrelated [2]. In this case, the autocorrelation matrix of the matched filter output $\mathbf{y}$ in (6), (9), or (11) is given by

$$
\mathbf{R}=E\left[\mathbf{y y}^{H}\right]=\left|\alpha_{0}\right|^{2} \mathbf{a}\left(\theta_{0}\right) \mathbf{a}^{\mathrm{H}}\left(\theta_{0}\right)+\mathbf{R}_{\mathrm{d}}
$$


where $\mathbf{R}_{\mathrm{d}}$ denotes the covariance matrix of the disturbance signal $\mathbf{d}=\sum_{i} \alpha_{i} \mathbf{a}\left(\theta_{i}\right)+\mathbf{n}$. Due to the different structures of the received signal, beamforming is different for the TS-MIMO from that for the other two, which is discussed separately.

\subsubsection{Phased-Array and Omni-MIMO}

The phased-array and omni-MIMO can obtain additional DOF by DC processing. Specifically, we vectorize the correlation matrix $\mathbf{R}$ as follows:

$$
\begin{aligned}
\mathbf{r}=\operatorname{vec}(\mathbf{R}) & =\left|\alpha_{0}\right|^{2}\left[\mathbf{a}^{*}\left(\theta_{0}\right) \otimes \mathbf{a}\left(\theta_{0}\right)\right] \\
& +\sum_{i=1}^{I}\left|\alpha_{i}\right|^{2}\left[\mathbf{a}^{*}\left(\theta_{i}\right) \otimes \mathbf{a}\left(\theta_{i}\right)\right]+\sigma_{n}^{2} \mathbf{e}
\end{aligned}
$$

where $\mathbf{e}=\left[\begin{array}{lll}\mathbf{e}_{1}^{T} & \cdots & \mathbf{e}_{L}^{T}\end{array}\right]^{T}$ with $\mathbf{e}_{i}^{T}$ being a column vector of all zeros except a 1 at the $i$ th position. The corresponding $L$ for the phased-array and omni-MIMO are $N^{2}$ and $N^{4}$, respectively. Note that the DC manifold $\mathbf{a}^{*}\left(\theta_{0}\right) \otimes \mathbf{a}\left(\theta_{0}\right)$ is a vector with redundancy. After removing the repeated elements and sorting, we get a filled virtual array with $N_{\text {syst }}$ distinct elements, which denotes the dimension of the DC manifold for the phased-array and omni-MIMO, given by

$$
\begin{aligned}
& N_{\mathrm{pa}}=2 K(J+1)-1 \\
& N_{\text {od }}=4 K(J+1)-3 .
\end{aligned}
$$

Applying the same redundancy removal and sorting procedure to $\mathbf{r}$ yield

$$
\mathbf{x}=\left|\alpha_{0}\right|^{2} \mathbf{u}\left(\theta_{0}\right)+\mathbf{v}
$$

where $\mathbf{u}\left(\theta_{0}\right)$ and $\mathbf{v}$ are the corresponding $N_{\text {syst }} \times 1$ virtual array steering vector and disturbance signal which includes contributions from the interference and the e vector. Note that the equivalent source signal vector $\mathbf{p}=\left[\begin{array}{llll}\left|\alpha_{0}\right|^{2} & \left|\alpha_{1}\right|^{2} & \ldots & \left|\alpha_{I}\right|^{2}\end{array}\right]$ for the coarray in (14), consists of the powers of the target and interferences. Spatial smoothing can be used to form a covariance matrix by dividing the $N_{\text {syst }}$ element DC into $\left(N_{\text {syst }}+1\right) / 2$ subarrays, each with $\left(N_{\text {syst }}+1\right) / 2$ elements. We denote the observation vector of the $i$ th subarray by $\mathbf{r}_{i}$. The spatial smoothed covariance matrix $\mathbf{R}_{\mathrm{ss}}$ can be obtained by (see [14] for further detail):

$$
\mathbf{R}_{\mathrm{ss}}=\frac{1}{\left(N_{\mathrm{syst}}+1\right) / 2} \sum_{i=1}^{\left(N_{\mathrm{syst}}+1\right) / 2} \mathbf{r}_{i} \mathbf{r}_{i}^{H}
$$

Using the subarray with elements at $m d, m=0,1, \cdots,\left(N_{\text {syst }}-1\right) / 2$, as the reference subarray, whose steering vector we denote as $\mathbf{u}_{1}\left(\theta_{0}\right)$, the minimum-variance distortionless response (MVDR) beamformer can be derived as

$$
\mathbf{w}\left(\theta_{0}\right)=\frac{\mathbf{R}_{\mathrm{SS}}^{-1} \mathbf{u}_{1}\left(\theta_{0}\right)}{\mathbf{u}_{1}^{\mathrm{H}}\left(\theta_{0}\right) \mathbf{R}_{\mathrm{SS}}^{-1} \mathbf{u}_{1}\left(\theta_{0}\right)}
$$




\subsubsection{TS-MIMO with Unequal Subarrays}

Note that the virtual array manifold $\mathbf{a}_{\mathrm{ts}}(\theta)$ in $(7)$ is not a Vandermonde vector, implying the virtual array of the TS-MIMO is non-uniform. Hence, DC processing and spatial smoothing are inapplicable. The MVDR beamformer can be implemented by directly using the covariance matrix (13), which leads to

$$
\mathbf{w}\left(\theta_{0}\right)=\frac{\mathbf{R}^{-1} \mathbf{a}_{\mathrm{ts}}\left(\theta_{0}\right)}{\mathbf{a}_{\mathrm{ts}}^{\mathrm{H}}\left(\theta_{0}\right) \mathbf{R}^{-1} \mathbf{a}_{\mathrm{ts}}\left(\theta_{0}\right)} .
$$

\subsubsection{Adaptive Estimation and DOF}

In practice, the covariance matrix $\mathbf{R}$ is unavailable. For adaptive implementation, the sample covariance matrix

$$
\hat{\mathbf{R}}=\frac{1}{T} \sum_{t=1}^{T} \mathbf{y}_{t} \mathbf{y}_{t}^{H}
$$

can be used in (14), where $\left\{\mathbf{y}_{t}\right\}_{t=1}^{T}$ are signal-free homogeneous training data collected from the range bins adjacent to the range bin of interest. By assuming a homogeneous environment, the training data are supposed to share similar interference characteristics as the test signal [1]. In practice, training data is usually limited when the radar operates in a non-homogeneous (e.g., urban, dense target) environment.

For the TS-MIMO, the observation $\mathbf{y}$ is filtered by the weight vector $\mathbf{w}$. The output SINR can be expressed as

$$
\gamma=\frac{\left|\alpha_{0}\right|^{2}\left[\mathbf{a}_{\mathrm{ts}}^{\mathrm{H}}\left(\theta_{0}\right) \hat{\mathbf{R}}^{-1} \mathbf{a}_{\mathrm{ts}}\left(\theta_{0}\right)\right]^{2}}{\mathbf{a}_{\mathrm{ts}}^{\mathrm{H}}\left(\theta_{0}\right) \hat{\mathbf{R}}^{-1} \mathbf{R} \hat{\mathbf{R}}^{-1} \mathbf{a}_{\mathrm{ts}}\left(\theta_{0}\right)}
$$

Similarly, for the phased-array and omni-MIMO, we have

$$
\gamma=\frac{\left|\alpha_{0}\right|^{4}\left[\mathbf{u}_{1}^{\mathrm{H}}\left(\theta_{0}\right) \hat{\mathbf{R}}_{s s}^{-1} \mathbf{u}_{1}\left(\theta_{0}\right)\right]^{2}}{\mathbf{u}_{1}^{\mathrm{H}}\left(\theta_{0}\right) \hat{\mathbf{R}}_{s s}^{-1} \tilde{\mathbf{R}} \hat{\mathbf{R}}_{s s}^{-1} \mathbf{u}_{1}\left(\theta_{0}\right)}
$$

where $\tilde{\mathbf{R}}$ denotes the covariance matrix of the disturbance signal after DC processing and spatial smoothing.

Now consider the DOF of the 3 transmit schemes. For the omni-MIMO or phased array, the virtual array as represented by (17) is a filled ULA with $N_{\text {syst }}$ elements. However, their DOF is decreased by spatial smoothing. Specifically, with $\mathbf{r}_{i}$ of size $\left[\left(N_{\text {syst }}+1\right) / 2\right] \times 1$ used in spatial smoothing, the DOF reduces to $\left(N_{\text {syst }}+1\right) / 2$. That is,

$$
\begin{gathered}
\mathrm{DOF}_{\mathrm{od}}=2 K(J+1)-1 . \\
\mathrm{DOF}_{\mathrm{pa}}=K(J+1) .
\end{gathered}
$$


The DOF of TS-MIMO is upper and lower bounded by (24) and (25). Nevertheless, as shown in Section 4, the TS-MIMO provides a better trade-off between the DOF and convergence when $T$, the number training signals used to estimate the covariance matrix $\hat{\mathbf{R}}$ [see (21)], is limited. The phasedarray and omni-MIMO require spatial smoothing and, in fact, they need estimate the 4th moment of the observed signal. As a result, they converge much more slowly than the TS-MIMO.

\section{Numerical Results}

To illustrate the benefits of using NA in the proposed transmit schemes, namely, the phased-array, omni-MIMO and TS-MIMO, we compare their application in the following array configurations: (a) ULA for both transmit (TX) and receive (RX); (b) ULA for TX and, NA for RX; (c) NA for both TX and RX. For each configuration, we have $N=4$ antennas with half wavelength separation between two adjacent elements for both the ULA and the inner array of the NA. The TS-MIMO employs 3 equal transmit subarrays for Configurations (a) and (b). Meanwhile, for Configuration (c), the TS-MIMO uses 5 unequal subarrays as described in Fig. 1. Configuration (a) is basically what was considered in [9]. Note that in the following simulations, DC processing is employed only for phased-array and omni-MIMO in Configuration (c); with ULA for TX as in (a) and (b), it can be shown that DC processing cannot improve the DOF for the omni-MIMO. For all 3 configurations, the received signal contains a target located at direction $10^{\circ}$. The target power, interference power and the noise variance are equal to $0.1,1$ and 0.01 , respectively.

First, we consider a case with $I=5$ interferences located at directions $\left[-30^{\circ},-15^{\circ}, 0^{\circ}, 20^{\circ}, 30^{\circ}\right.$. Fig. 2 depicts the mean output SINR versus the number of training, which are calculated as (22) for TS-MIMO, and (23) for phased-array/omni-MIMO. With sufficient training, higher SINRs can be achieved by each method in Figs. 2(b) and 2(c) than those in Fig. 2(a), indicating that all schemes benefit from using NA. It is noted that the performances of omni-MIMO and TS-MIMO are similar with ULA for TX and NA for RX. This is because they have similar degrees of freedom (DOF). In Configuration (b), the DOF is 8 for TS-MIMO and 9 for omni-MIMO. Therefore, both of them are able to suppress $I=5$ interferences in Fig. 2(b). However, neither of them can handle $I \geq 9$ interferences, which is the case considered in the next example. It is also seen from Fig. 2(c) that the TS-MIMO converges faster and achieves higher SINR than the phased-array and omni-MIMO for the considered training size. This is because the latter employ spatial smoothing to estimate the 4 -th moment of the observed signal, which led to the lower convergence.

We next increase $I=9$ interferences located at directions $\left[-60^{\circ},-45^{\circ},-30^{\circ},-15^{\circ}, 0^{\circ}, 20^{\circ}, 30^{\circ}\right.$, 
$45^{\circ}, 60^{\circ}$. The mean output SINR and beampatterns are shown in Fig. 3 and Fig. 4 . It is seen that Configurations (a) and (b) now fail with negative SINR because $I$ has exceeded their capability. Meanwhile, Configuration (c) employs NA for both receive and transmit, which affords additional DOF, allowing the omni-MIMO and TS-MIMO to adequately mitigate the interferences and achieve notable improvement in their SINR. In particular, consider the omni-MIMO in Configurations (b) and (c). It is easy to show that the DOF for Configuration (b) is $K(J+1)+N-1$, while the DOF for Configuration (c) is given by (24). Plugging in the simulation parameters, the DOFs are 9 and 11, respectively. Therefore, Configuration (b) has fewer DOF and cannot handle all the 9 interference. In turn, the output SINR for (b) is significantly degraded. On the other hand, Configuration (c) achieves a good output SINR because it can adequately mitigate all 9 interferences. It is also seen that for small to moderate training size $(T<1000)$, the TS-MIMO has the highest SINR due to its faster convergence. With sufficient training, the omni-MIMO is able to surpass the TS-MIMO, due to more DOF. Fig. 4 depicts the beampatterns of the different schemes with $T=500$, where the vertical dashed lines indicate the locations of the interferences. It is seen that only the TS-MIMO is able to place nulls at all interference locations, corroborating its higher SINR shown in Fig. 3(c). We next show the benefits of using NAs in direction of arrival (DOA) estimation for a case of $D=9$ sources located at directions $\left[-60^{\circ},-45^{\circ},-30^{\circ},-15^{\circ}, 0^{\circ}, 20^{\circ}, 30^{\circ}, 45^{\circ}, 60^{\circ}\right]$ with identical power of 1 in the presence of white noise with zero mean and variance 0.01. Fig. 5 depicts the spectrum obtained by using MUSIC estimator applied to all configurations except the phased-array ${ }^{1}$ with $T=100$. Clearly, only the proposed TS-MIMO and omni-MIMO in (c) can resolve all 9 sources. It is also observed that the peak locations and amplitudes of the omni-MIMO spectrum in Configuration (c) are inaccurate for some sources. This is due to the fact that $T=100$ is small for omni-MIMO, which is unable to attain convergence. Then we compare the DOA estimation accuracy in Configuration (c) in terms of the root mean squared error (RMSE), defined as

$$
\operatorname{RMSE}=\frac{1}{D} \sum_{d=1}^{D} \sqrt{\frac{1}{Q} \sum_{q=1}^{Q}\left(\hat{\theta}_{d}^{(q)}-\theta_{d}\right)^{2}}
$$

where $Q$ is the number of Monte Carlo runs and $\hat{\theta}_{d}^{(q)}$ is the estimate of the $d$ th target $\theta_{d}$ at the $q$ th trial. Fig. 6 shows the performance of the two methods versus the number of training signals $T$, averaged over $Q=100$ Monte Carlo runs. It is seen that TS-MIMO converges fast and exhibits better precision than omni-MIMO.

\footnotetext{
${ }^{1}$ Note that the plot of phased-array is not shown in Fig. 5, due to the fact that the number of targets exceeds the dimension of the phased-array covariance matrix, which results in a null noise subspace. As a result, the MUSIC estimator cannot be applied.
} 


\section{Conclusion}

We investigated three transmit schemes for MIMO radars with a colocated NA. A novel transmit subaperturing approach with unequal subarrays was proposed. Phased-array and omni-MIMO were also extended to the NA scenario. TS-MIMO was shown to outperform the other methods when training is limited, while omni-MIMO with difference coarray processing achieved the highest output SINR with sufficient training.

\section{References}

[1] J. Li and P. Stoica, MIMO Radar Signal Processing. New York: Wiley, 2009.

[2] A. M. Haimovich, R. S. Blum, and L. J. Cimini, "MIMO radar with widely separated antennas," IEEE Signal Process. Mag., vol. 25, no. 1, pp. 116-129, Jan. 2008.

[3] R. Niu, R. S. Blum, P. K. Varshney, and A. L. Drozd, "Target localization and tracking in noncoherent multiple-input multiple-output radar systems," IEEE Trans. Aerosp. Electron. Syst, vol. 48, no. 2, pp. 1466-1489, Apr. 2012.

[4] W.-Q. Wang, "Phased-MIMO radar with frequency diversity for range-dependent beamforming," IEEE Sensors J., vol. 13, no. 4, pp. 1320-1328, Apr. 2013.

[5] W. Zhang, W. Liu, J. Wang, and S. Wu, "Joint transmission and reception diversity smoothing for direction finding of coherent targets in MIMO radar," IEEE J. Sel. Topics Signal Process., vol. 8, no. 1, pp. 115-124, Feb. 2014.

[6] S. Ahmed and M.-S. Alouini, "MIMO radar transmit beampattern design without synthesising the covariance matrix," IEEE Trans. Signal Process., vol. 62, no. 9, pp. 2278-2289, May 2014.

[7] J. Xu, G. Liao, S. Zhu, L. Huang, and H. C. So, "Joint range and angle estimation using MIMO radar with frequency diverse array," IEEE Trans. Signal Process., vol. 63, no. 13, pp. 3396-3410, Jul. 2015.

[8] S. Imani, S. A. Ghorashi, and M. Bolhasani, "SINR maximization in colocated MIMO radars using transmit covariance matrix," Signal Process., vol. 119, pp. 128-135, 2016.

[9] H. Li and B. Himed, "Transmit subaperturing for MIMO radars with co-located antennas," IEEE J. Sel. Topics Signal Process., vol. 4, no. 1, pp. 55-65, Feb. 2010.

[10] A. Hassanien and S. A. Vorobyov, "Phased-MIMO radar: A tradeoff between phased-array and MIMO radars," IEEE Trans. Signal Proces., vol. 58, no. 6, pp. 3137-3151, Jun. 2010.

[11] A. Hassanien and S. A. Vorobyov, "Transmit energy focusing for DOA estimation in MIMO radar with colocated antennas," IEEE Trans. Signal Process., vol. 59, no. 6, pp. 2669-2682, 2011.

[12] W. Khan, I. M. Qureshi, A. Basit, and M. Zubair, "Hybrid phased MIMO radar with unequal subarrays," IEEE Antennas Propag. Lett., vol. 14, pp. 1702-1705, 2015.

[13] W.-Q. Wang and H. C. So, "Transmit subaperturing for range and angle estimation in frequency diverse array radar," IEEE Trans. Signal Proces., vol. 62, no. 8, pp. 2000-2011, Apr. 2014.

[14] P. Pal and P. Vaidyanathan, "Nested arrays: a novel approach to array processing with enhanced degrees of freedom," IEEE Trans. Signal Process., vol. 58, no. 8, pp. 4167-4181, Aug. 2010.

[15] K. Han and A. Nehorai, "Nested vector-sensor array processing via tensor modeling," IEEE Trans. Signal Process., vol. 62, no. 10, pp. 2542-2553, May 2014.

[16] K. Han, P. Yang, and A. Nehorai, "Calibrating nested sensor arrays with model errors," IEEE Trans. Antennas Propag., vol. 63, no. 11, pp. 4739-4748, Nov. 2015.

[17] C. Zhu, H. Chen, and H. Shao, "Joint phased-MIMO and nested-array beamforming for increased degreesof-freedom," Int. J. Antennas Propag., vol. 2015, no. 989517, pp. 1-11, 2015. 


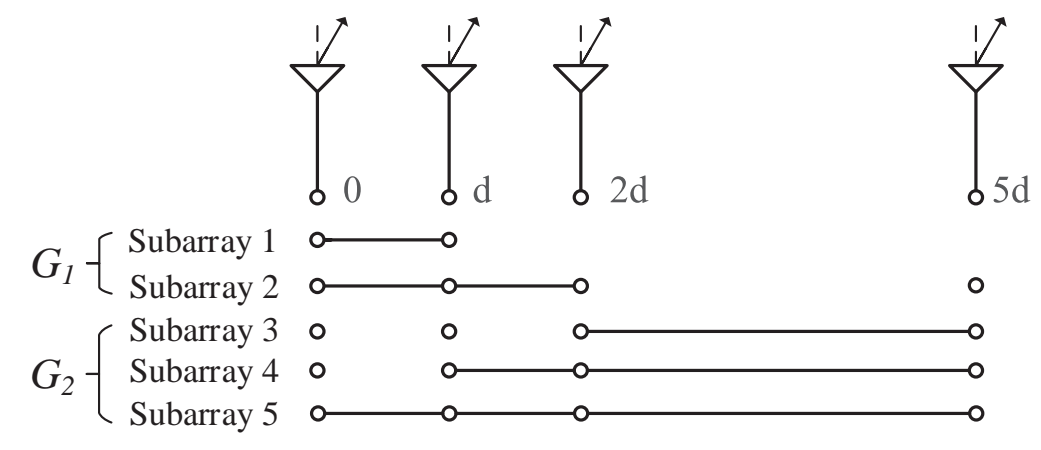

Figure 1: Proposed TS scheme with $N=4$ antennas. 
(a)

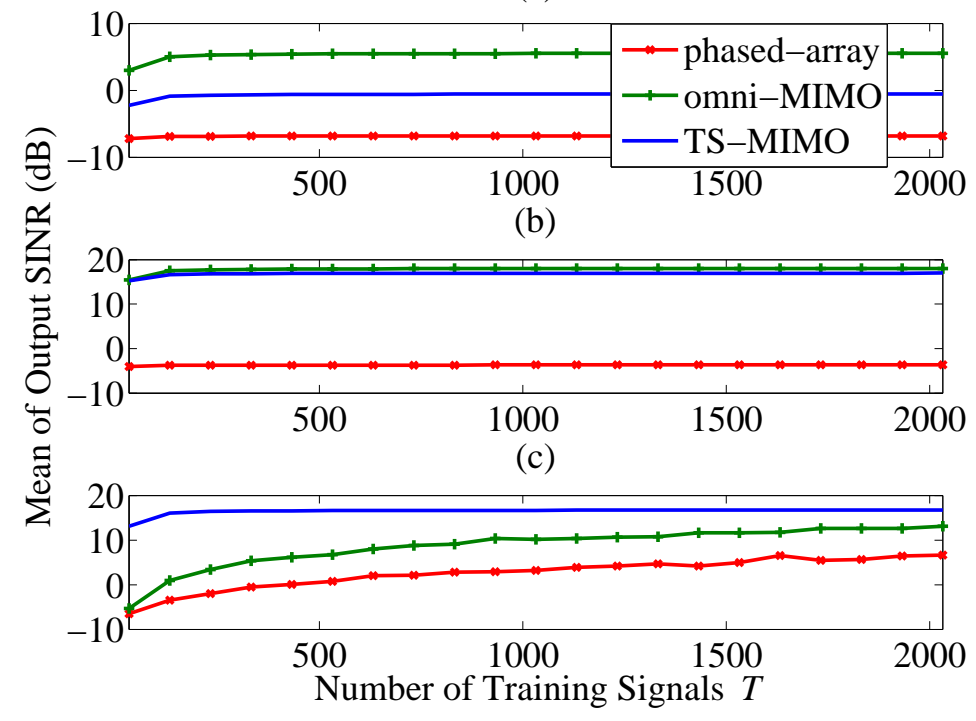

Figure 2: Mean output SINR with $I=5$ interferences. (a) ULA for both TX and RX, (b) ULA for TX and NA for RX, (c) NA for both TX and RX. 
(a)

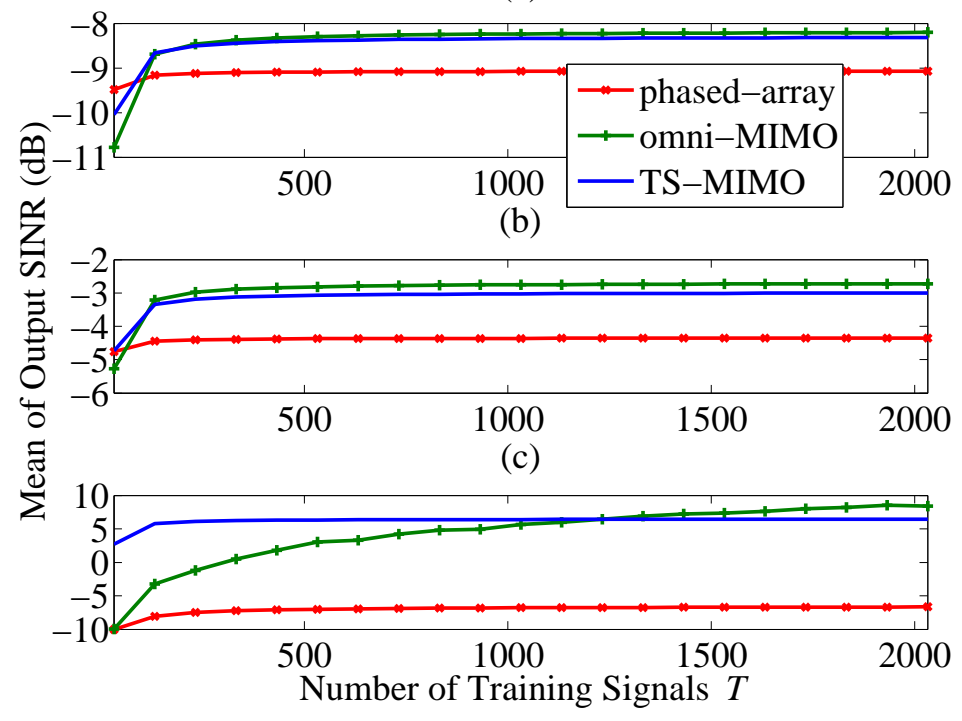

Figure 3: Mean output SINR with $I=9$ interferences. (a) ULA for both TX and RX; (b) ULA for TX and NA for RX; (c) NA for both TX and RX. 
(a)
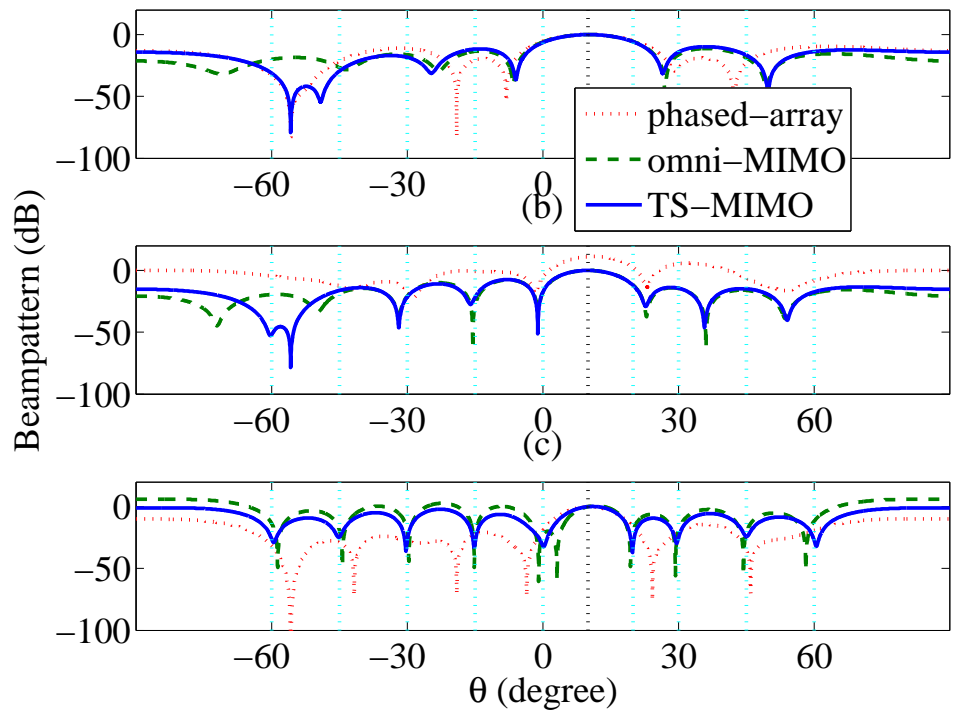

Figure 4: Beampatterns with $I=9$ and $T=500$ training signals. (a) ULA for both TX and RX; (b) ULA for TX and NA for RX; (c) NA for both TX and RX. 
(a)

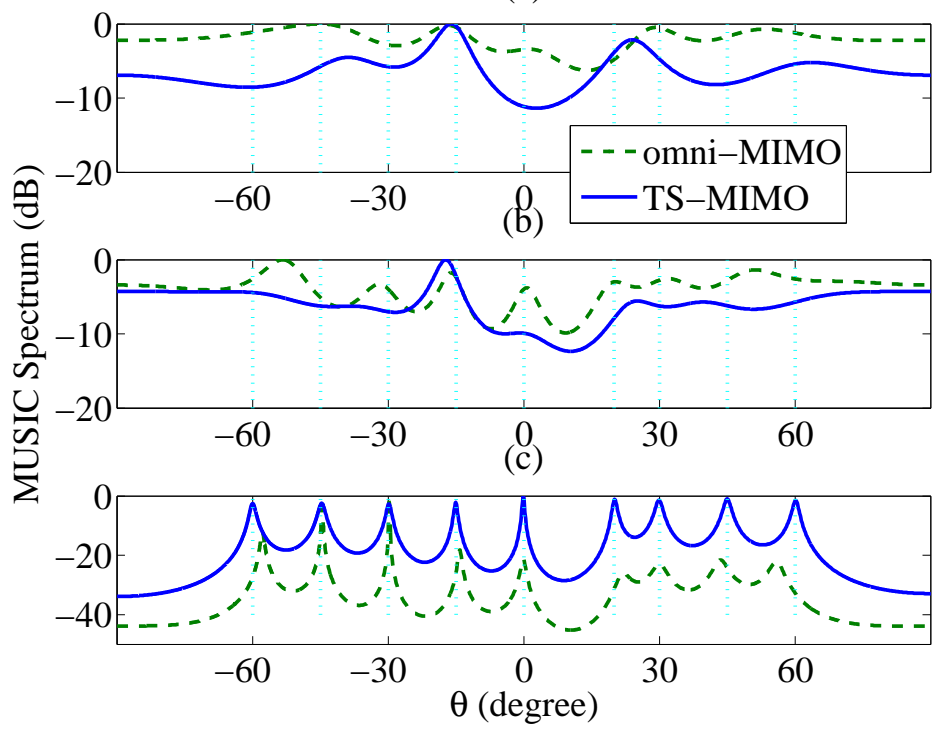

Figure 5: Spatial spectra as a function of $\theta$ with 9 sources and $T=100$ training signals. (a) ULA for both TX and RX; (b) ULA for TX and NA for RX; (c) NA for both TX and RX. 


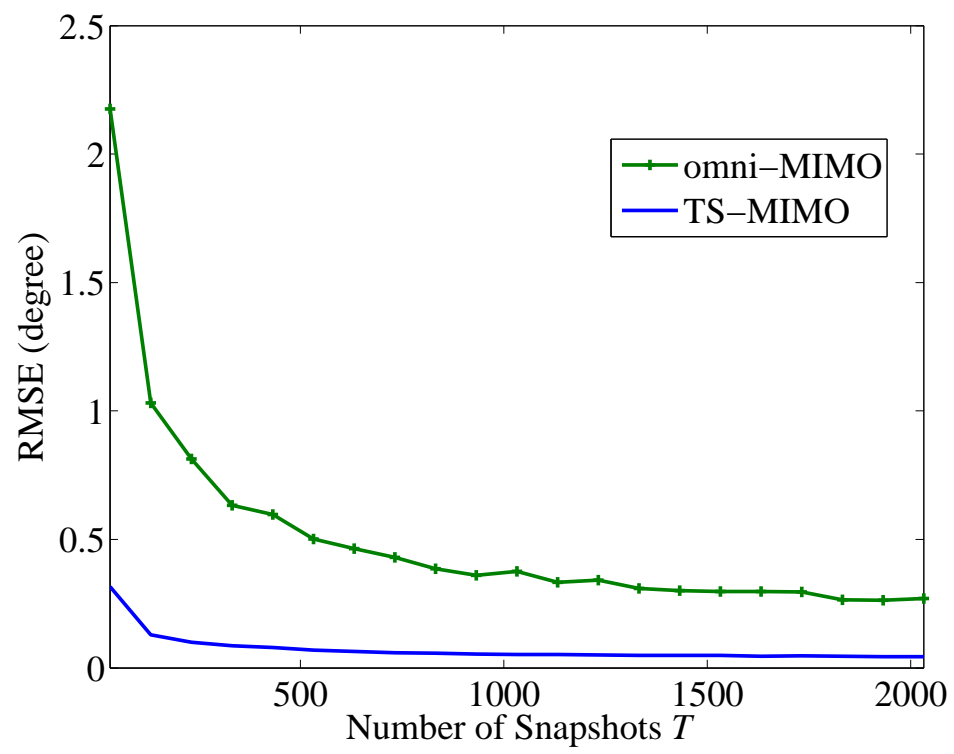

Figure 6: RMSE versus the training size $T$ with $D=9$ sources. 